\title{
ÜBER QUADRATISCHE CHARAKTERSUMMEN
}

\author{
TOMIO KUBO'TA
}

Es gibt zwei verschiedene Arten von Summen, die gewöhnlich Gausssche Summen genannt werden. Die eine Summe wird von einer Zahl $\omega \neq 0$ eines algebraischen Zahlkörpers $F$ bestimmt. Sei nämlich o die Differente von $F$ und sei $\omega \delta=\frac{\mathfrak{b}}{\mathfrak{a}}$ mit zueinander primen, ganzen Idealen $\mathfrak{a}, \mathfrak{b}$ von $F$. Dann hat die Summe die Gestalt

$$
C(\omega)=\sum_{\mu \bmod \alpha .} e\left(\omega \mu^{2}\right), \quad(\mu: \operatorname{ganz}),
$$

wo

$$
\boldsymbol{e}(u)=e^{2 \pi i S_{F / \mathbf{Q}^{u}}} \quad \text { für } u \in F
$$

ist, und dabei $\mathbf{Q}$ bzw. $S$ der rationale Zahlkörper bzw. die absolute Spur bedeutet. Eine grundlegende Forschung dieser Summen findet man etwa in Hecke [3].

Die andere Summe wird dagegen von einem Kongruenzcharakter $\%$ der Idealgruppe von $F$ bestimmt. Es sei $\mathfrak{i}$ der (endliche) Führer von $\%$ und $\beta$ eine totalpositive Zahl von $F$, für die die Beziehung

$$
\frac{\mathfrak{h}}{\delta \mathfrak{f}_{x}}=(\beta), \quad\left(\mathfrak{h}, \mathfrak{f}_{x}\right)=1,
$$

mit einem ganzen Ideal $\mathfrak{h}$ von $F$ besteht. Die in Rede stehende Summe ist dann

$$
\tau(\chi)=\chi(\mathfrak{h}) \sum_{z \operatorname{mo:1} \mathfrak{f}_{x}} \chi(z) e(\beta z), \quad\left(z: \mathfrak{f}_{x} \text {-ganz, totalpositiv }\right),
$$

wo $\%(z)$ statt $\chi((z))$ steht und $\chi(z)=0$ für $\left(z, f_{x}\right) \neq 1$ ist. Auch trat diese Summe in die Arbeiten Heckes bei der Funktionalgleichung der L-Reihen ein (vgl. Hasse [1]). Nach Hasse [2] bekommt die Summe (2) eine andere Form

$$
\tau(\chi)=\sum_{x \bmod b-1} \%(x) e(x)
$$

wo $x$ ein vollkommenes, totalpositives Restsystem von $\left(\delta f_{x}\right)^{-1} / \delta^{-1}$ durchläuft

Received May 19, 1961. 
und

$$
(x)=\frac{\mathfrak{x}}{\mathfrak{D} \mathfrak{f}_{x}}, \quad\left(\mathfrak{x}, \mathfrak{f}_{\mathrm{x}}\right)=1
$$

mit einem ganzen Ideal $\mathfrak{x}$ von $F$ ist. Setzen wir $x=\beta z$ in (3), so erhalten wir sofort den Ausdruck in (2), und also erkennen, dass (2) von $\beta$ unabhängig ist.

Um die eben erklärten zwei Summen sich von einander unterscheiden $z u$ lassen, soll im folgenden die erste Summe die Exponentialsumme heissen, die zweite dagegen die Charaktersumme, während man die beiden solchen Summen meistens als gleich anzusehen pflegt. Gewiss haben sie viele Ähnlichkeiten. Zum Beispiel gibt es zwei bemerkenswerte Tatsachen in bezug auf diese Summen, die aus einer und derselben analytischen Quelle entspringen. Nämlich ist einerseits das Reziprozitätsgesetz eine wichtige Eigenschaft von $C(\omega)$ [3]. Andererseits, wenn $a$ die Anzahl der unendlichen Verzweigungsprimstellen von $\chi$ ist und man

$$
W(\chi)=\frac{(-i)^{a}}{\sqrt{N \mathfrak{F}_{\chi}}} \tau(\chi)
$$

setzt, so gilt die bekannte Formel

$$
\prod_{\chi} W(\chi)=1
$$

wo $\chi$ alle zu einem abelschen Erweiterungsköper $K / F$ zugeordneten Kongruenzcharaktere von $F$ durchläuft. Ist also insbesondere $\%$ quadratisch, i.e. $\chi^{2}=1$, so ergibt sich

$$
W(\chi)=1 .
$$

Diese und jene arithmetischen Folgerungen, die entweder mit $C(\omega)$ oder $W(\chi)$ zusammenhängen, gewinnt man in der Tat, indem man die Transformations. formel der Thetafunktion gebraucht.

Es ist aber zu bemerken, dass die Exponential- und Charaktersummen nicht völlig identish, sondern von etwas verschiedener Natur sind. Dazu sei vorerst erwähnt, dass $C(\omega)$ nicht immer in der Form $\tau(\chi)$ mit $\chi^{2}=1$ geschrieben wird. Noch wesentlicher ist jedoch, dass das Reziprozitätsgesetz der Exponentialsummen, obwohl sehr verwandt aussieht, nicht ganz gleichbedeutend mit (5) für quadratischen $\chi$ ist, denn das Reziprozitätsgesetz folgt allein aus der Transformationsformel der Thetafunktion, während (5) mit $\chi^{2}=1$ erst dann am 
klarsten hervorgeht, wenn zusätzlich das Zerlegungsgesetz der relativ-quadratischen Körper angenommen wird [2]. Ferner hat das Reziprozitätsgesetz der Exponentialsummen das quadratische Reziprozitätsgesetz für Potenzreste zur Folge [3], aber keine entsprechende Tatsache für (5) ist selbstverständlich.

Das Ziel der vorliegenden Arbeit ist die Verschiedenheit beider Summen in einigen Einzelheiten $z u$ betrachten. Nachdem wir in $\S 1$ eine einfache Beziehung zwischen $C(\omega)$ und $\tau(\%)\left(\chi^{2}=1\right)$ in einem Spezialfall angesehen habe, wollen wir in $\$ 2$ und $\S 3$ den Zusammenhang der Charaktersummen mit dem quadratischen Reziprozitätsgesetz untersuchen, der ziemlich anders ausfällt als bei den Exponentialsummen. Beispielsweise werden diese Ergebnisse im Fall des rationalen Körpers in $\S 4$ dargestellt.

\section{§ 1. Übersicht über den Zusammenhang zwischen Exponential- und Charaktersummen.}

Es sei $a \neq 0$ ein $z$ u 2 primes ganzes Ideal von eines algebraischen Zahlkör. pers $F$ und sei vorausgesetzt, dass $\mathfrak{a}=p_{1} p_{2} \cdots p_{r}$ ein Produkt von lauter verschiedenen Primidealen $\mathfrak{p}_{i}$ ist. Man nehme ferner ein $z \mathfrak{u}$ a primes ganzes Ideal $\mathfrak{c}_{:}$von $F$ derart, dass $\mathfrak{p}_{i} \mathfrak{c}_{i}=\left(\pi_{i}\right)\left(\pi_{i} \in F\right)$ ein Hauptideal ist, und setze $\pi=\Pi_{\pi_{i}}$, $\mathfrak{c}=\Pi \mathfrak{c}_{\mathfrak{i}}$. Ist dann $\mathfrak{b}$ die Differente von $F$ und ist $\mathfrak{b}$ ein $z \mathfrak{u} \mathfrak{a}$ primes ganzes Ideal in der Idealklasse von $b a$, so ist für die mit

$$
(\omega)=\frac{\mathfrak{b}}{\mathfrak{d} \mathfrak{a}}, \quad \omega \in F
$$

definierte Summe $C(\omega)$ in (1) ein einfacher Zusammenhang mit den Charaktersummen $z u$ finden. Angenommen im allgemein, dass $a=a_{1} a_{2}$ mit zueinander primen ganzen Idealen $a_{1}, \mathfrak{a}_{2}$ von $F$ ist und dass $a_{1} \mathfrak{n}_{1}=\left(\alpha_{1}\right), a_{2} \mathfrak{n}_{2}=\left(\alpha_{2}\right) \quad\left(\alpha_{1}\right.$ $\left.\alpha_{2} \in F\right)$ mit ganzen, $z u$ a primen Idealen $\mathfrak{n}_{1}, \mathfrak{n}_{2}$ von $F$ zutrifft, so ist nach Hecke [3]

$$
C(\omega)=\left(\frac{\alpha_{2}}{a_{1}}\right)\left(\frac{\alpha_{1}}{a_{2}}\right) C\left(\alpha_{2} \omega\right) C\left(\alpha_{1} \omega\right) .
$$

In unserem Fall gilt also induktiv

$$
C(\omega)=\prod_{i \neq \jmath}\left(\frac{\pi_{j}}{\mathfrak{p}_{i}}\right) \cdot \prod_{i} C\left(\pi \pi_{i}^{-1} \omega\right)
$$

Ist daher $C_{i}(\omega)=\left(\frac{\pi i}{a_{i}^{-1}}\right) C\left(\omega_{1}\right)$ und ist $\omega_{i}=\pi \pi^{-1} \omega$, so ergibt sich 


$$
C(\omega)=\prod_{i=1}^{r} C_{i}(\omega)
$$

was eine Komponentenzerlegung von $C(\omega)$ ist. Da $\mathfrak{\not}_{i}$ prim ist, wird $C\left(\omega_{i}\right)$ nach Hecke [3] in die Charaktersumme

$$
C\left(\omega_{i}\right)=\sum_{\substack{\mu \text { mod } \\ \mu \neq 0\left(p_{i}\right)}}\left(\frac{\mu}{p_{i}}\right) e\left(\omega_{i} / \mu\right) \quad(\mu: \operatorname{gan} z)
$$

transformiert. Es sei hier überdies vorausgesetzt, dass $\mathfrak{a}=\mathfrak{f}_{x}$ der Führer eines quadratischen Kongruenzcharakters von $F$ ist. Die mit diesem $\psi$ definierte Charaktersumme $\tau(\%)$ in (2) ist dann zerlegbar in ihre kanonischen lokalen Komponenten (vgl. etwa Hasse [2]). Es gibt nämlich für beliebigen Kongruenzcharakter \% von $F$ die Zerlegung

$$
\begin{aligned}
& \tau(\%)=\prod_{p \text { endl. }} \tau_{p}^{\prime}(\psi)
\end{aligned}
$$

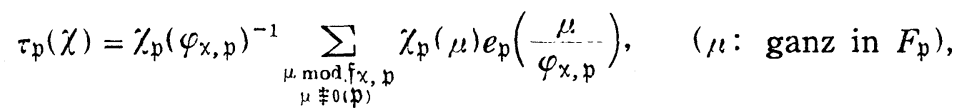

wo $\%$ die mittels Idelen definierte $p$-Komponente von $\%, e_{\mathfrak{p}}(u)=e^{2 \pi i S_{\mathfrak{p}} u}$ mit lokaler Spur $S_{\mathfrak{p}}, \varphi_{x, \mathfrak{p}}$ eine Zahl im p-adischen Körper $F_{\mathfrak{p}}$, die den $\mathfrak{p}$-Bestandteil

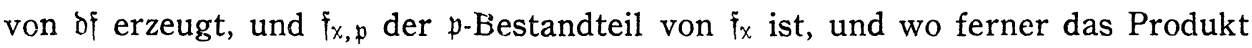
in (8) alle endliche Primstellen durchläuft.

Ist nun $\%$ quadratisch, so ist $\chi_{\mathfrak{p}_{i}}(u)=\left(\frac{u}{\mathfrak{p}_{i}}\right)$. Da aber $\left(\omega_{i}\right)=\left(\pi \pi_{i}^{-1} \omega\right)$ $=\frac{\mathfrak{c} c_{i}^{-1} \mathfrak{b}}{\mathfrak{b} \mathfrak{p}_{i}}$ ist, mag $\omega_{i}^{-1}$ als $\varphi_{x, \mathfrak{p}_{i}}$ genommen werden, wenn nur $\mathfrak{b}, \mathfrak{c}$ auch zu $b$ prim sind. Sind weiter $\mathfrak{p}_{i}^{\prime}, \mathfrak{p}_{i}^{\prime \prime}, \ldots$ die Konjugierten von $\mathfrak{p}_{i}$, so ist

$$
S(u)=S_{p_{i}}(u)+S_{p^{\prime} i}(u)+S_{p^{\prime \prime} i}(u)+\cdots \cdot
$$

Da der Nenner von $\triangleright \omega_{i}$ nicht durch $\mathfrak{p}_{i}^{\prime}, \mathfrak{p}_{i}^{\prime \prime}, \ldots$ teilbar ist, sind $S_{\mathfrak{p}^{\prime}}\left(\omega_{i} / !\right)$, $S_{p^{\prime \prime} i}\left(\omega_{i} / \ell\right), \ldots$ sämtlich ganz, und daher

$$
\left.e^{(} \omega_{i} \mu\right)=e_{p_{i}}\left(\omega_{i}, \ell\right) \text {. }
$$

Aus (9) und (10) folgt also

$$
\tau_{\mathfrak{p}_{i}}\left(\chi_{i}\right)=\chi_{\mathfrak{p}_{i}}\left(\omega_{i}\right) \sum_{\substack{\mu \bmod \mathfrak{p}_{i} \\ \mu \neq\left(\mathfrak{p}_{i}\right)}}\left(\frac{\mu}{\mathfrak{p}_{i}}\right) e\left(\omega_{i} \mu\right), \quad(\mu: \operatorname{ganz})
$$

Setzen wir weiterhin $\pi_{i}$ als totalpositiv voraus, so folgt nach (7), (11), dass 


$$
\begin{aligned}
& \%_{p_{i}}(\pi \omega) C_{i}(\omega)=\psi_{p_{i}}\left(\omega_{i}\right) \%_{p_{i}}\left(\pi_{i}\right) C_{i}(\omega)=\psi_{p_{i}}(\omega) \cdot \prod_{\mathfrak{p}+a} \psi_{p}\left(\pi_{i}\right)^{-1} \cdot\left(\frac{\pi_{i}^{-1}}{a p_{i}^{-1}}\right) C_{i}(\omega) \\
& =\%\left(c_{i}\right) \psi_{p_{i}}\left(\omega_{i}\right) C\left(\omega_{i}\right)=\%\left(c_{i}\right) \tau_{\mathfrak{p}_{i}}(\chi)
\end{aligned}
$$

ist, wo das Produkt alle zu a prime endliche Primstellen durchläuft. Diese Formel gilt für $p_{1}, p_{2}, \ldots, p_{r}$. Für sonstige endliche Primstellen gilt aber $\tau_{\mathfrak{p}}(\%)=\chi\left(D_{\mathfrak{p}}\right)$ nach $(9)$, wo $\grave{D}_{\mathfrak{p}}$ der $\mathfrak{p}$-Bestandteil von $\mathfrak{b}$ ist. Ist ferner $\mathfrak{b}_{\mathfrak{p}}$ bzw. $\mathfrak{c}_{\mathfrak{p}}$ der $\mathfrak{p}$-Bestandteil von $\mathfrak{b}$ bzw. $\mathfrak{c}$, so ist $\frac{\mathfrak{b}_{\mathfrak{p}} \mathfrak{c}_{\mathfrak{p}}}{\mathfrak{D}_{\mathfrak{p}}}$ der von $\pi \omega$. Setzt man also $C_{\mathfrak{p}_{i}}(\omega)$ $=C_{i}(\omega), c^{\mathfrak{p}_{i}}=c_{i}$ für $\mathfrak{p}_{1}, \mathfrak{p}_{2}, \ldots, \mathfrak{p}_{r}$ und $C_{\mathfrak{p}}(\omega)=1, \mathfrak{c}^{\mathfrak{p}}=1$ für sonstige $\mathfrak{p}$, so ist

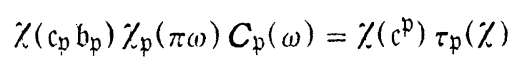

für jede endliche Primstelle von $F$. Einfachheit halber sei jetzt (1) totalpositiv. Multipliziert man dann (12) für alle endliche $\mathfrak{p}$, und beachtet (6) und (8), so ergibt sich die Beziehung

$$
\chi(b) C(\omega)=\tau(\%)
$$

die zwischen Exponential- und Charaktersummen besteht, wo, vorsichtshalber wiederholt, $\mathfrak{a}$ der Führer von $\%, \omega \mathfrak{b}=\frac{\mathfrak{b}}{\mathfrak{a}}$ mit $(\mathfrak{a}, \mathfrak{b})=(\mathfrak{b}, \mathfrak{b})=1, \omega$ totalpositiv, und a ein Produkt verschiedener, zu 2 primer Primideale von $F$ ist.

\section{§. Reziprozitätsgesetze, die aus $W(\%)=1$ folgen.}

$\mathrm{Da}$ (5) nichts anders als die Vorzeichenbestimmung der Gaussschen Summen ist, ist es wahrscheinlich, dass (5) zu einem gewissen Reziprozitätsgesetz führt. Ein so zu erhaltenes Reziprozitätsgesetz ist aber etwas komplizierter Natur. Denn, wie schon in der Einleitung bemerkt wurde, muss dabei ausser der Funktionalgleichung der L-Funktion noch das Zerlegungsgesetz der Klassenkörpertheorie angewandt werden, um einen Beweis von (5) im allgemeinen Fall $z u$ gewinnen. Hier soll übrigens untersucht werden, was für ein Reziprozitätsgesetz wir elementar erhalten können, indem wir nur (5) annehmen. Dazu genügt eine Rechnung eines Faktorensystems. Es seien $\%, \varkappa^{\prime}$ Kongruenzcharaktere von $F, \mathfrak{f}_{x}, \mathfrak{f}_{x}$, ihre Führer, und sei $\left(\mathfrak{f}_{x}, \mathfrak{f}_{x}\right)=1$. Man nehme totalpositive $\beta, \beta^{\prime}, \theta \in F$ mit

$$
(\beta)=\frac{\mathfrak{h}}{\mathfrak{d} \mathfrak{f}_{x}}, \quad\left(\beta^{\prime}\right)=\frac{\mathfrak{h}^{\prime}}{\mathfrak{d} \mathfrak{f}_{x^{\prime}}}, \quad\left(\mathfrak{h}, \mathfrak{f}_{x}\right)=\left(\mathfrak{h}^{\prime}, \mathfrak{f}_{x^{\prime}}\right)=1
$$

und 


$$
(\theta)=\frac{\mathfrak{w}}{\delta \mathfrak{f}_{x} \mathfrak{f}_{x^{\prime}}}, \quad\left(\mathfrak{w}, \mathfrak{f}_{x} \mathfrak{f}_{x^{\prime}}\right)=1,
$$

wo $\mathfrak{h}, \mathfrak{h}^{\prime}$ und $\mathfrak{w}$ ganze Ideale von $F$ bedeuten. Dann ist $\mathfrak{f}_{x x}=\mathfrak{f}_{x} \mathfrak{f}_{x}$, und

$$
w=\frac{\beta z+\beta^{\prime} z^{\prime}}{\theta}
$$

durchläuft ein totalpositives, vollkommenes Restsystem mod $f_{x x^{\prime}}$, falls $z$ bzw. $z^{\prime}$ ein totalpositives, vollkommenes Restsystem mod $\mathfrak{f}_{x}$ bzw. $\bmod f_{x}$, durchläuft. Andererseits folgt aus

$$
\begin{aligned}
& \chi \chi^{\prime}(w)=\chi \chi \chi^{\prime}\left(\frac{\beta}{\theta} z+\frac{\beta^{\prime}}{\theta} z^{\prime}\right)=\chi\left(\frac{\beta}{\theta} z+\frac{\beta^{\prime}}{\theta} z^{\prime}\right) \chi^{\prime}\left(\frac{\beta}{\theta} z+\frac{\beta^{\prime}}{\theta} z^{\prime}\right) \\
& =\chi\left(\frac{\beta}{\theta} z\left(1+\frac{\beta^{\prime}}{\theta} \frac{z^{\prime}}{z}\right)\right) \chi^{\prime}\left(\frac{\beta^{\prime}}{\theta} z^{\prime}\left(1+\frac{\beta}{\beta^{\prime}} \frac{z}{z^{\prime}}\right)\right)=\chi\left(\frac{\beta}{\theta} z\right) \chi^{\prime}\left(\frac{\beta^{\prime}}{\theta} z^{\prime}\right),
\end{aligned}
$$

dass

$$
\chi \chi^{\prime}(\mathfrak{w} w)=\chi\left(\mathfrak{G} \mathfrak{F}_{X}, z\right) \chi^{\prime}\left(\mathfrak{h}^{\prime} \mathfrak{f}_{x} z^{\prime}\right)
$$

ist. Also ergibt sich

$$
\tau(\chi) \tau\left(\chi^{\prime}\right)=\sum_{z} \sum_{z^{\prime}} \chi(\mathfrak{h} z) \chi^{\prime}\left(\mathfrak{h}^{\prime} z^{\prime}\right) e(\theta w)=\chi\left(\mathfrak{f}_{x^{\prime}}\right)^{-1} \chi^{\prime}\left(\mathfrak{f}_{x}\right)^{-1} \tau\left(\chi \chi^{\prime}\right)
$$

oder die folgende Faktorensystemrelation

$$
\frac{\tau(\chi) \tau\left(\chi^{\prime}\right)}{\tau\left(\chi \chi^{\prime}\right)}=\chi\left(\mathfrak{f}_{x^{\prime}}\right)^{-1} \chi^{\prime}\left(\mathfrak{f}_{x}\right)^{-1}
$$

Sind nun $\chi$ und $\chi^{\prime}$ quadratisch und ist $a, a^{\prime}$ bzw. $a^{\prime \prime}$ die Anzahl der unendlichen Verzweigungsprimstellen von $\chi, \chi^{\prime}$ bzw. $\chi \chi^{\prime}$, so gilt nach (4), (5) und (14) die Formel

$$
\chi\left(\mathfrak{F}_{x^{\prime}}\right) \chi^{\prime}\left(\mathfrak{f}_{\mathrm{x}}\right)=i^{a^{\prime \prime}-a-a^{\prime}},
$$

die eine verlangte Reziprozität ist. Natürlich sind (14) und (15) richtig, wenn $\chi$ oder $\chi^{\prime}$ auch trivial ist.

Noch eine Reziprozitätsrelation folgt aus (5), der die Komponentenzerlegung von $\tau(\chi)$ zugrunde liegt. Es sei nämlich $\sigma_{p}$ der Quotient der in (9) auftretenden Summe nach ihrem absoluten Betrag. Dann ist $\tau_{\mathfrak{p}}(\chi)=\chi_{\mathfrak{p}}\left(\varphi_{x, \mathfrak{p}}\right)^{-1} \sigma_{\mathfrak{p}}\left|\tau_{\mathfrak{p}}(\chi)\right|$, und, wenn $\chi$ quadratisch ist, so folgt

$$
\prod_{p \in n i l,} \chi_{p}\left(\varphi_{x, p}\right)=i^{-a} \prod_{p \in n d 1,} \sigma_{p}
$$


aus (5), wo a die gleiche Bedeutung wie in (4) hat. Der Inhalt der Formeln (15), (16) soll noch im folgenden untersucht werden.

\section{§ 3. Reziprozitätsgesetze mit einer gewöhnlichen Form.}

In diesem Abschnitt wollen wir versuchen, die Bedeutung der Formel (15) noch klarer zu machen, indem wir aus (15) überhaupt eine Reziprozitätsrelation zwischen Zahlen entnehmen. Wird z.B. der Charakter $\%$ bzw. $\chi^{\prime}$ in (15) als der der Erweiterung $F(\sqrt{ } a)$ bzw. $F\left(\sqrt{ } a^{\prime}\right)\left(a, a^{\prime} \in F\right)$ zugeordneten Kongruenzcharakter festgesetzt, so wird wohl ein quadratisches Reziprozitätsgesetz gewonnen. Solch eine Reziprozität ist aber insofern vom geringeren Sinn, als dabei ausser (15) noch einmal die Klassenkörpertheorie gebraucht wird. Diesen Umstand zu vermeiden und eine nur aus (15) stammende Folgerung zu erreichen, soll man etwas anders verfahren. Wir wollen hier nur eine einfache Methode darlegen.

Es sei $\lambda$ ein Homomorphismus der Idealgruppe eines algebraischen Zahlkörpers $F$ in die Multiplikationsgruppe der Zahlen eines algebraischen Zahlkörpers $L$, wo $\lambda$, wie bei Kongruenzcharakteren, auf der Gruppe von der $z u$ einem geeigneten Ideal peimen Idealen beschränkt werden mag. Die im folgenden vorkommenden Ideale oder Zahlen sind, wenn erforderlich, auch einer ähnlichen Bedingung $z u$ unterwerfen. Für $\lambda$ sei noch vorausgesetzt, dass man für jedes ganze Ideal $m$ von $L$ ein ganzes Ideal $\mathfrak{f}$ von $F$ derart bestimmen kann, dass $\operatorname{man} \lambda((\alpha))=1(\bmod \mathfrak{m})$ hat, falls $\alpha \in F$ totalpositiv und $\alpha \equiv 1(\bmod \mathfrak{j})$ ist. Bei sogenannten Grössencharakteren vom Typus $A_{0}$ (Weil [6]) sind die Bedingungen von $\lambda$ sicher der Fall. Nun sei in ein Ideal von $L$ und sei

$$
\%(\mathfrak{a})=\left(\frac{\lambda(\mathfrak{a})}{\mathfrak{m}}\right)
$$

mit dem quadratischen Restsymbol in $L$. Dann ist $\chi$ ein quadratischer Kongruenzcharakter von $F$. Für ein anderes Ideal $\mathrm{m}^{\prime}$ von $L$ werde gleichartig ein $\chi^{\prime}$ definiert. Sind dann die Führer $\mathfrak{f}_{x}, f_{x}$, von $\chi, \chi^{\prime}$ zueinander prim, und setzt man $\mu=\lambda\left(\mathfrak{f}_{x}\right), \mu^{\prime}=\lambda\left(\mathfrak{f}_{x^{\prime}}\right)$, so folgt aus (15) die Beziehung

$$
\left(\begin{array}{c}
k^{\prime} \\
\mathrm{m}
\end{array}\right)\left(\frac{\mu}{\mathrm{m}^{\prime}}\right)=i^{a^{\prime \prime}-a-a^{\prime}},
$$

die ein Reziprozitätsgesetz mit einer gewöhnlichen Form ist. 


\section{$\S 4$. Der rationale Fall.}

Um die bisher erhaltenen Ergebnisse noch wirklicher darzustellen, werden sie hier im rationalen Zahlkörper $\mathbf{Q}$ betrachtet. Ein Homomorphismus $\lambda$ in $\S \mathbf{3}$ wird von einer Abbildung gegeben, die einem Ideal von $\mathbf{Q}$ sein positives erzeugendes Element zuordnet. Dabei ist also $F=L=\mathbf{Q}$ und $\lambda((a))=|a|$. Sind $m$, $m^{\prime}$ zueinander prime, ungerade, quadratfreie natürliche Zahlen, so ist $m$ bzw. $\boldsymbol{m}^{\prime}$ der (endliche) Führer von $\chi((a))=\left(\frac{\lambda((a))}{m}\right)$ bzw. $\chi^{\prime}((a))=\left(\frac{\lambda((a))}{m^{\prime}}\right)$, und $p_{\infty}$ ist eine oder keine Verzweigungsprimstelle von $\chi$ bzw. $\chi^{\prime}$, je nachdem $m$ bzw. $m^{\prime} \equiv 1(\bmod 4)$ oder $\equiv-1(\bmod 4)$ ist. Nach $(17)$ ist daher

$$
\left(\frac{m^{\prime}}{m}\right)\left(\frac{m}{m^{\prime}}\right)=(-1)^{(m-1) / 2 \cdot\left(m^{\prime}-1\right) / 2}
$$

Also folgt das Reziprozitätzgesetz für quadratische Reste im rationalen Körper direkt aus der Formel (5).

Nun wollen wir die Formel (16) von $\S 2$ aufnehmen. Es sei $m$ wie oben eine ungerade, quadratfreie natürliche Zahl und sei $\chi((a))=\left(\frac{\lambda((a))}{m}\right)=\left(\frac{|a|}{m}\right)$. Ist dann $m=p_{1} p_{2} \cdots p_{r}$ die Primzahlzerlegung von $m$, so kann $\varphi_{x, p_{i}}=p_{i}$ in (9) gesetzt werden. Ist ferner $\chi_{p_{i}}$ die $p_{i}$-Komponente von $\chi$, so gilt

$$
\chi_{p_{i}}(u)=\left(\frac{u}{p_{i}}\right)
$$

für eine ganzrationale Zahl $u$ mit $\left(u, p_{i}\right)=1$. Folglich ist

$$
\chi_{p_{i}}\left(p_{i}\right)^{-1}=\prod_{j \neq i} \chi_{p_{i}}\left(p_{i}\right)=\prod_{j \neq i}\left(\frac{p_{i}}{p_{j}}\right)=\left(\frac{p_{i}}{m_{i}}\right)
$$

mit $m_{i}=\frac{m}{p_{i}}$, und die Relationen (18) und (19) setzen umgekehrt den Charakter $\chi_{p_{i}}$ fest. Jetzt stimmt nach (18) die in (9) auftretende Summe mit

$$
\tau\left(\varkappa_{i}\right)=\sum_{\substack{u \text { mod } \\ u \neq 0\left(p_{i}\right)}}\left(\frac{u}{p_{i}}\right) e\left(\frac{u}{p_{i}}\right)
$$

überein, wo $\chi_{i}((a))=\left(\frac{|a|}{p_{i}}\right)$ ist, und $\sigma_{\mathfrak{p}}$ in (16) wird wegen $W\left(\chi_{i}\right)=1$ durch

$$
\sigma_{p_{i}}= \begin{cases}1 & p_{i} \equiv 1(\bmod 4) \\ i & p_{i} \equiv-1(\bmod 4)\end{cases}
$$

bestimmt. Ist also $a_{i}=0$ oder 1 , je nachdem $p_{i} \equiv 1$ oder $-1(\bmod 4)$, und 
$a=0$ oder 1 , je nachdem $m \equiv 1$ oder $-1(\bmod 4)$, so ergibt sich aus (16) die Formel

$$
\prod_{i}\left(\frac{p_{i}}{m_{i}}\right)=i^{a-\Sigma a_{i}}
$$

von der eine Bedeutung von (16) abzulesen ist. Falls $m=p_{1} p_{2}$ ist, so wird (20) wieder ein gewöhnliches Reziprozitätsgesetz. Eine mit (16) verwandte Formel für Exponentialsummen hat Gauss auf seinen veirten Beweis des quadratischen Reziprozitätsgesetzes verwendet (vgl. etwa Reichardt [5]). Wenn umgekehrt eine Formel wie (16) oder (20) vorausgesetzt wird, so bestimmt sich eine Charaktersumme durch lokale Charaktersummen. Man sieht ferner, dass im rationalen Fall die Formel (16) mit der Formel (15) gleichbedeutend ist, wenn wie oben $\gamma((a))=\left(\frac{|a|}{m}\right)$ mit $m=p_{1} p_{2}$ betrachtet wird. Aber solche zwei Arten von Formeln sollen im allgemeinen unterschieden werden, denn eine lokale Charaktersumme kann nicht immer als eine Summe mit einem Kongruenzcharakter angesehen werden.

Die in $\S 1$ bewiesene Beziehung zwischen Exponential- und Charaktersummen bekommt im rationalen Fall eine bekannte, einfache Gestalt. Es sei wieder $\chi((a))=\left(\frac{|a|}{m}\right)$ mit einer quadratfreien, ungeraden natürlichen Zahl $m$. Da dann $m$ der Führer von $\%$ ist, kann man $\omega^{-1}=\mathfrak{a}=m, \delta=\mathfrak{b}=1$ in (13) setzen und die Resultate von $\S 1$ anwenden. Nänlich ergibt sich $C(m)=\tau(\%)$ nach (13), und

$$
\sum_{u \bmod m} e\left(\begin{array}{c}
u^{2} \\
m
\end{array}\right)=\sum_{u \bmod m} \chi(u) e\left(\begin{array}{c}
u \\
m
\end{array}\right)
$$

nach (1) und (2). Wenn wir ferner $c=1, \pi \omega=1$ in (12) setzen, so erkennen wir, dass in diesem Fall die in $\$ 1$ eingeführten Komponentenzerlegungen von $C(\omega)$ und $\tau(\%)$ miteinander identisch sind.

Es werde beiläufeg erwähnt, dass der hierbei benutzte Charakter $\%$ dem quadratischen Körper $F=\mathbf{Q}\left(\sqrt{m^{*}}\right)$ mit $m^{*}=(-1)^{(m-1 / 2} m$ entspricht, und also $\left(\frac{*, m^{*}}{p}\right)$ die $p$-Komponente von $\%$ ist, was aber in vorliegender Arbeit entbehrlich ist.

\section{§. Bemerkungen.}

Es sei $\%$ ein quadratischer Kongruenzcharakter eines algebraischen Zahlkörpers $F$ und $L(s, \%)$ sei die von $\%$ bestimmte Heckesche $L$-Funktion. Ist 
dann $M(s)$ das Produkt von $L(s, \%)$ mit passenden Gammafaktoren, so gilt die Funktionalgleichung

$$
M(s, \chi)=W(\not) M(1-s, \not)
$$

$\mathrm{Da} M(s, \chi)$ für einen reellen Wert von $s$ reell ist, ergibt sich

$$
M\left(\frac{1}{2}+i t, \%\right)=W(\%) M\left(\frac{1}{2}-i t, \%\right) \quad(t: \text { reell })
$$

oder

$$
M\left(\frac{1}{2}+i t, \chi\right)=W(\chi) M\left(\frac{1}{2}+i t\right)
$$

Daher ist (5) gleichbedeutend mit der Aussage, dass $M$ für $s=\frac{1}{2}+i t$ reell ist. Dazu reicht $M\left(\frac{1}{2}\right) \neq 0$, oder auch $L\left(\frac{1}{2}, \chi\right) \neq 0$ aus. Könnte man diese Behauptungen direkt bestätigen, so würde man (5) und die Ergebnisse von $\S 2$, $\S 3$ rein analytisch erreichen. Ein solches Verfahren scheint jedoch ziemlich schwer zu sein.

Fasst man (5) dagegen als eine Folge von der Funktionalgleichung der $L$ hien und dem Zerlegungsgesetz der Klassenkörpertheorie, so stellt der Gedankengang von $\S 2, \S 3$ eine Analogie der Hey-Zornschen Theorie [7], die aus der Funktionalgleichung der Heyschen Zetafunktion und dem Zerlegungsgesetz in Quaternionenalgebren das quadratische Reziprozitätsgesetz in einem beliebigen algebraischen Zahlkörper folgen lässt.

Es seien schliesslich $\%, \chi^{\prime}$ zwei quadratische Kongruenzcharaktere von $F$. Es sei $w_{\mathfrak{p}}(\chi)=\frac{\tau_{\mathfrak{p}}(\chi)}{\left|\tau_{\mathfrak{p}}(\chi)\right|}$ und

$$
\frac{w_{\mathfrak{p}}(\chi) w_{\mathfrak{p}}\left(\chi^{\prime}\right)}{w_{\mathfrak{p}}\left(\chi \chi_{\chi^{\prime}}\right)}=\left(\frac{\chi, \chi^{\prime}}{\mathfrak{p}}\right) \quad(\mathfrak{p}: \text { endlich })
$$

mit in (8) definierten lokalen Komponenten von $\tau\left(\chi_{\infty}\right), \tau\left(\chi^{\prime}\right)$. Ferner sei $\left(\frac{\chi, \chi^{\prime}}{p_{\infty}}\right)$ $=-1$ oder 1 für eine unendliche Primstelle $\mathfrak{p}_{\infty}$, je nachdem $\mathfrak{p}_{\infty}$ eine gemeinsame Verzweigungsprimstelle von $\chi, \chi^{\prime}$ ist oder nicht. Dann ergibt sich nach (5), dass

$$
\prod_{\mathfrak{p}}\left(\frac{\not, \not^{\prime}}{\mathfrak{p}}\right)=1
$$

ist, wo $\mathfrak{p}$ alle Primstellen von $F$ durchläuft. Wenn die (endlichen) Führer von 
$\chi, \chi^{\prime}$ zueinander prim sind, so ist (15) eine leichte Folge von (21). Däuber hinaus entspricht (21) der Produktformel des Normenrestsymbols, wie es in [4] zu sehen ist.

\section{LITERATURVERZEICHNIS}

[1] H. Hasse, Bericht I (1926), Ia (1927), II (1930), Jahresbericht der D.M.V.

[2] H. Hasse, Artinsche Führer, Artinsche $L$-Funktionen und Gausssche Summen über endlich-algebraischen Zahlkörpern, Acta Salmanticensia, Universidad de Salamanca (1954).

[3] E. Hecke, Vorlesungen über die Theorie der algebraischen Zahlen, Leipzig 1923.

[4] T. Kubota, Local relation of Gauss sums, Acta Arithmetica 6 (1960), 285-294.

[5] H. Reichardt, C. F. Gauss Gedenkband anlässlich des 100.Todestages am 23.Februar 1955, Leipzig 1957.

[6] A. Weil, On a certain type of characters of the idèle-class group of an algebraic number field, Proc. Int. Symp. Alg. Number Theory, Tokyo-Nikko 1955, 1-7.

[7] M. Zorn, Note zur analytischen hyperkomplexen Zahlentheorie, Abh. Math. Sem. Hamburg 9 (1933), 197-201.

Mathematisches Institut,

Universität zu Nagoya 